\title{
Increased Risk of Atherosclerosis in Patients with Sarcoidosis
}

\author{
Elena Bargagli ${ }^{\mathrm{a}}$ Elisabetta Rosi ${ }^{\mathrm{a}}$ Massimo Pistolesi $^{\mathrm{a}} \quad$ Federico Lavorini ${ }^{\mathrm{a}}$ \\ Luca Voltolini $^{b}$ Paola Rottolic \\ a Section of Respiratory Medicine, Department of Clinical and Experimental Medicine, Department of Clinical and \\ Experimental Biomedical Sciences, University of Florence, and b Thoracic Surgery Unit, University Hospital Careggi, \\ Florence, and ${ }^{\mathrm{C}}$ Respiratory Medicine Department of Clinical and Immunological Sciences, Siena University, \\ Siena, Italy
}

\section{Keywords}

Sarcoidosis · Atherosclerosis · Lipid metabolism

\section{Abstract}

Sarcoidosis is a systemic granulomatous disease of unknown etiology. Recent studies demonstrated that its pathogenesis is related with enhanced oxidative stress (protein carbonylation and lipid peroxidation) and alterations in the circulating lipid profile. Alterations of lipid metabolism (including the reduction in high-density lipoprotein cholesterol levels and apolipoprotein A1 concentrations) induce plasma membrane, bronchial and lung capillary endothelial cell damage in sarcoidosis patients. Dyslipidemia is associated with increased oxidative stress, diminished overall antioxidative protection and increased risk for atherosclerosis. Very recently increased cardiovascular biomarkers (in particular alterations of lipoprotein A and d-dimer concentrations) were observed in sarcoidosis patients, mainly in those with a high risk of atherosclerosis. Chitotriosidase, a biomarker of sarcoidosis activity and macrophage activation, is increased in serum and bronchoalveolar lavage fluid of patients with sarcoidosis as well as in patients with atherosclerosis. Lipidomics and other recent methodologies allowed the discovery of proteins involved in lipid metabolism and sarcoidosis
\end{abstract}

\section{KARGER}

(C) 2017 S. Karger AG, Basel

E-Mail karger@karger.com

www.karger.com/pat pathogenesis, such as serum amyloid A, a biomarker of sarcoidosis activity, involved in innate immune response, inflammation and apolipoprotein metabolism. In this review lipid metabolism alteration and atherosclerosis risk in sarcoidosis patients were discussed in order to contribute to this novel and interesting research topic.

(c) 2017 S. Karger AG, Basel

\section{Introduction}

Sarcoidosis is a systemic granulomatous disease of unknown origin characterized by accumulation of activated proliferating $\mathrm{T}$ lymphocytes and mononuclear phagocytes in affected organs $[1,2]$. Interaction between antigens and antigen-presenting cells polarizes $\mathrm{T}$ lymphocytes to the $\mathrm{T}$ helper 1 phenotype, followed by lymphocyte, macrophage, epithelioid and giant cell recruitment, proliferation, activation, and differentiation, determining formation of granulomas [1-3].

The clinical expression, natural history and prognosis of sarcoidosis are unpredictable, and no reliable indicators of clinical outcome are available. Some patients experience spontaneous remission and others develop chronic persistent disease, sometimes with relapses and

Elena Bargagli, $\mathrm{MD}, \mathrm{PhD}$

Section of Respiratory Medicine, Department of Clinical and Experimental Medicine Department of Clinical and Experimental Biomedical Sciences, University of Florence Largo Brambilla 3, IT-50134 Florence (Italy)

E-Mail bargagli2@gmail.com 
different responses to treatment $[1,2]$. Acute sarcoidosis is associated with Lofgren syndrome while chronic sarcoidosis has insidious onset and slow progression with large variations between individuals and almost invariable lung involvement. Symptoms, if any, are usually few (dry cough, dyspnea, chest pain, and rarely a low fever). In progressive advanced forms with fibrotic lung involvement there may be severe hypoxemia and pulmonary hypertension [1-4].

The pathogenesis of sarcoidosis has been associated with activation of alveolar macrophages and $\mathrm{T}$ lymphocytes that release a broad spectrum of mediators, such as cytokines, enzymes and chemokines, that modulates granulomatous inflammation as well as lipid metabolism $[1,5]$. Changes in lipid metabolism have been shown to damage the plasma membrane [5], as well as bronchial and lung capillary endothelial cells. Sarcoidosis has recently been associated with an increased pulse wave index, which may indicate an early stage of atherosclerosis [6-10]. Enhanced production of reactive oxygen species and alterations in circulating lipid profile have been reported in this granulomatous disease [11, 12].

Oxidative stress and altered lipid metabolism are thought to play a role in the pathogenesis of sarcoidosis, although the significance of blood oxidative stress/antioxidant defense and alterations in lipid status parameters in sarcoidosis is unclear [13]. Interestingly, dyslipidemia has been associated with increased oxidative stress and diminished overall antioxidative protection but the potential role of changes in lipid metabolism in the pathogenesis of the disease has not been widely investigated [11-13]. In the last 15 years, certain authors have begun to investigate the proteins involved in lipid metabolism in sarcoidosis, finding a reduction in high-density lipoprotein (HDL) cholesterol levels and apolipoprotein A1 (apoA1) concentrations in patients with active disease. This suggested increased risk of atherosclerosis in sarcoidosis patients, irrespective of exposure to steroids [1418]. Lipidomics showed that several proteins involved in lipid metabolism, including serum amyloid A, are expressed differently in patients than in controls [19]. This protein, involved in innate immune response, inflammation, and apolipoprotein metabolism, proved to be a biomarker of sarcoidosis activity. It displaces apoA1 inducing HDL catabolism in sarcoidosis [20].

In this review we collect the last 10 years of literature on lipid metabolism and atherosclerosis risk in sarcoidosis patients with the aim to discuss their potential pathogenetic role in sarcoidosis.

Atherosclerosis in Sarcoidosis

\section{Lipid Metabolism and Alteration of Capillary Endothelial Cells}

Lipid metabolism alteration plays a role in the early phase of sarcoid formation $[4,5]$. It damages the plasma membrane and bronchial and lung capillary endothelial cells, as recently demonstrated by immunohistochemistry [5]. A role of lipid biology in the development of sarcoidosis is indicated by observations on endothelial cells and membrane proteins present in the surface of the endothelium in lung samples from patients with initialstage disease [5]. Capillary endothelial cells from patients showed droplets containing saturated and unsaturated fatty acids in the cytoplasm, as well as in the capillary lumen, suggesting that endothelial damage may precede granuloma formation [5].

Adipophilin has recently been described among the membrane proteins contained in these lipid droplets in sarcoidosis tissue [6]. Also known as perilipin 2, this interesting protein binds lipids with high affinity and is involved in liver fibrosis and lung cancer. It has recently been analyzed by immunohistochemical staining in sarcoidosis lung tissue. Altered protein expression with local, mainly intracellular, distribution in clustered histiocytes has been reported to be a typical feature of sarcoidosis [6].

Morphological evaluation of damage to respiratory tract capillary endothelial cells in patients with sarcoidosis also showed that lipid droplets exhibiting dark monophasic density (unsaturated fatty acids) were surrounded by abundant lysosomes in the cells [7]. In this granulomatous disease, biphasic lipid droplets with dark and lucent (saturated fatty acids) densities, accompanied by a few lysosomes containing the residual bodies of undigested lipid-containing substances, have been reported in respiratory tract biopsies [7]. The plasma membrane is frequently damaged in capillary endothelial cells containing biphasic lipid droplets and lucent monophasic droplets, as well as in those with dark monophasic droplets. Lipid droplets in respiratory tract capillary endothelial cells from sarcoidosis patients have been closely associated with mitochondrial alterations, including large number, mobilization, and remarkable morphological changes including swelling [7].

Considering the lipid modifications observed in endothelium of sarcoid lungs, the risk of atherosclerosis has recently been evaluated in these patients by angiogenic analysis and peripheral cardiovascular biomarker assay [8]. An altered ankle brachial index with respect to controls was reported irrespective of systemic treatment. In

Pathobiology 2017;84:258-263

DOI: $10.1159 / 000477736$ 
the same population increased cardiovascular biomarkers (especially peripheral concentrations of lipoprotein A and d-dimer) were demonstrated, and it was concluded that this granulomatous interstitial lung disease is characterized by an increased pulse wave index, which indicates early-stage atherosclerosis.

The increased risk of atherosclerosis in sarcoidosis patients was confirmed in another very recent publication. The study considered the subgroup of cardiac sarcoidosis patients and demonstrated that atherosclerosis was facilitated by upregulation of cholesterol absorption and downregulation of cholesterol synthesis [9].

\section{Oxidative Stress Associated with Dyslipidemia}

Reactive oxygen species released by phagocytes are involved in the pathogenesis of several diffuse lung diseases, including sarcoidosis. The crucial roles of nitrosyl-induced stress and lipid peroxidation in the pathogenesis of sarcoidosis have been reported by many authors [10-17] as well as higher concentrations of carbonylated proteins in bronchoalveolar lavage (BAL) fluid of sarcoidosis patients with respect to controls, indicative of the contribution of oxidatively modified proteins to the development of the disease $[15,16]$.

Among the markers of lipid peroxidation, 8-isoprostane has been analyzed in BAL fluid and exhaled breath condensate from patients with sarcoidosis [12] and was found to be higher with respect to controls. To validate the prognostic value of this marker, its concentrations in exhaled breath from patients with sarcoidosis were correlated with disease severity parameters. It was concluded that low initial 8-isoprostane concentrations may be a positive prognostic factor and that lipid peroxidation plays a crucial role in lung inflammation in active sarcoidosis [12]. More recently, 8-isoprostane levels were also measured in serum and BAL fluid from patients with sarcoidosis or idiopathic pulmonary fibrosis and controls. They were much higher in the former, sustaining the hypothesis that lipid peroxidation plays a major role in this granulomatous inflammatory disorder [14].

In 2012, a paper was published on oxidant-antioxidant balance and lipid metabolism in sarcoidosis [13]. Interestingly, increased oxidative stress burst (associated with increased malondialdehyde, superoxide anion, total oxidant status, prooxidant-antioxidant balance and triglyceride) and reduced antioxidant defense status (low superoxide dismutase activity and HDL cholesterol concentrations) were recorded in patients with sarcoidosis. Serum and erythrocyte malondialdehyde were the strongest predictors of disease, and elevated oxidative stress had higher clinical accuracy than lipid status abnormality [13]. It was clearly stated that oxidative stress and lipid status parameters altered with respect to controls indicated an increased risk of atherosclerosis.

\section{Lipidomics}

The "-omics" sciences (genomics, proteomics, metabolomics) collectively characterize and quantify a pool of biological molecules to obtain insights into the pathogenetic mechanisms, clinical phenotyping and prognosis of a specific disease. Lipidomics is based on large-scale studies of the lipid pathways and networks involved in a specific condition. The first "omic" applied to the study of sarcoidosis was proteomics that led to the identification of different protein pathways and profiles involved in its pathogenesis [16]. Classical 2-dimensional electrophoresis applied to the study of bronchoalveolar lavage showed that proteins involved in lipid metabolism were altered in sarcoidosis and involved in its development $[18,19]$.

In a recent proteomic study, various BAL proteins (such as plastin 2, fatty acid binding protein, annexin A2 and apoA1) involved in the regulation of lipid metabolism were found to be expressed differently in sarcoidosis patients and controls [19]. Proteins downregulated in sarcoidosis included plastin 2 and fatty acid-binding protein 4 . The latter regulates fatty acid metabolism and cholesterol trafficking and is expressed by adipocytes and macrophages. In sarcoidosis patients, increased LDL uptake and cholesterol efflux seem to be facilitated by a deficit in fatty acid binding protein 4 in alveolar macrophages $[18,19]$. Another protein involved in lipid metabolism and expressed differently in BAL fluid of sarcoidosis patients is apoA1. Its main function is to remove low-density lipoprotein (LDL) particles present in foamy macrophages, inhibiting expression of proinflammatory cytokines $[18,19]$. Anomalous lipid droplets in the respiratory tract of sarcoidosis patients may explain the protein's increase in BAL fluid [18, 19]. Increased serum concentrations of triglycerides are associated with high levels of zinc- $\alpha_{2}$ glycoprotein, involved in lipid presentation to CD4 cells [19]. Insulin resistance in sarcoidosis patients is mainly regulated by 2 specific proteins identified by a proteomic approach and elevated in BAL fluid of sarcoidosis patients: retinoid binding protein 4 and $\alpha_{2}$-HS-glycoprotein [19].
260

Pathobiology 2017;84:258-263

DOI: $10.1159 / 000477736$
Bargagli/Rosi/Pistolesi/Lavorini/

Voltolini/Rottoli 
Fig. 1. Proteins involved in lipid metabolism and atherosclerosis risk in sarcoidosis as assessed by lipidomics.

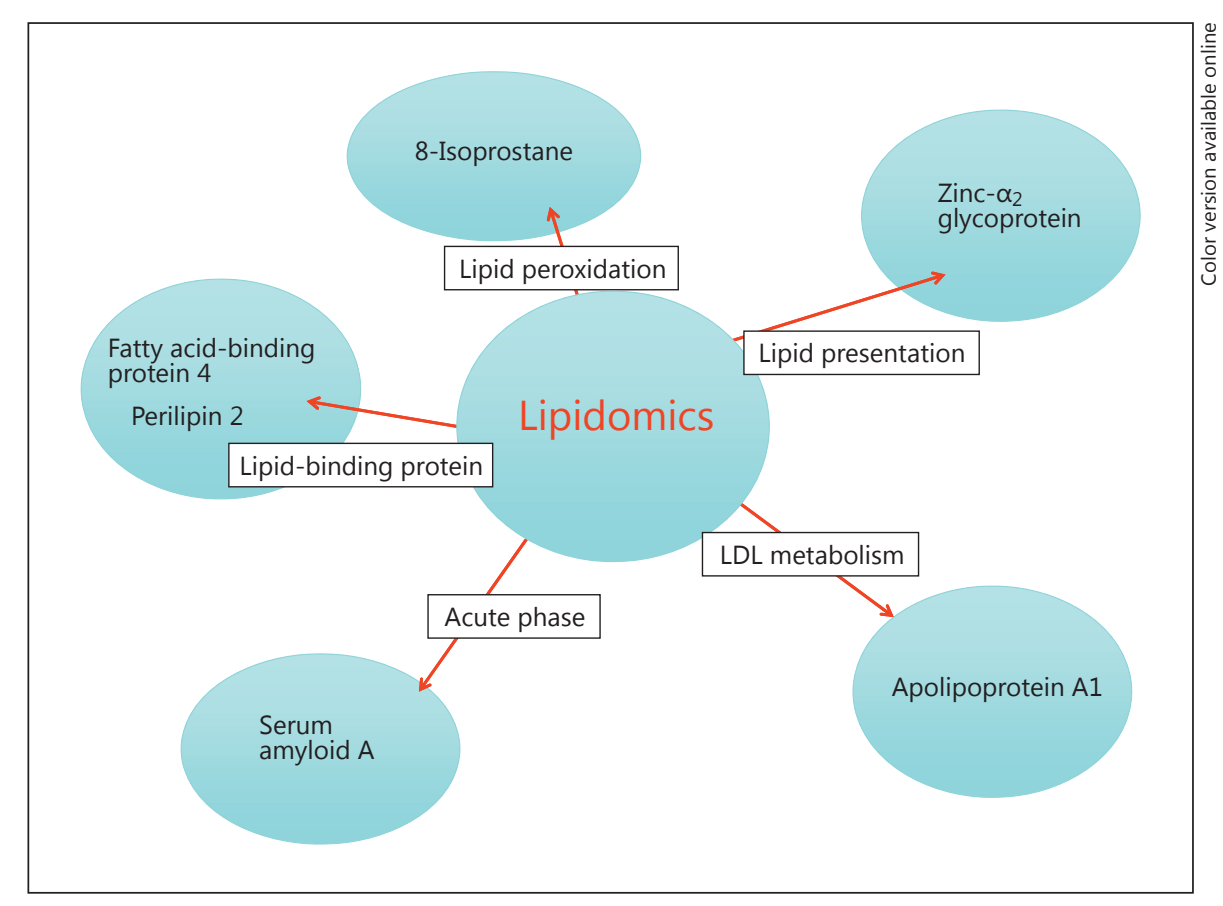

Different research groups have also used lipidomics to identify serum amyloid A (SAA) in BAL fluid and serum of patients with sarcoidosis, demonstrating that this acute phase protein is overexpressed and involved in disease pathogenesis [19-21]. Validation of the proteomic results by quantitative assays allowed the authors to demonstrate that SAA is a prognostic biomarker (being elevated in acute sarcoidosis with respect to inactive disease). It is also useful for ruling out sarcoidosis because it is only increased in sarcoidosis and not in other granulomatous lung diseases, such as tuberculosis [20,21].

Salazar et al. [22-24] demonstrated in many studies that SAA concentrations are significantly higher in active sarcoidosis patients than controls, especially in those with severe anomalies of lipid metabolism, such as low HDL cholesterol levels and apoA1 concentrations. SAA emerged as a key molecule regulating innate immune responses and inflammation in sarcoidosis through Tolllike receptor 2 [25]. It proved to be involved in apolipoprotein metabolism during inflammatory responses by displacing apoA 1 , inducing HDL catabolism or inhibiting lecithin-cholesterol acyltransferase activity [24]. The prognostic role of SAA as a biomarker of sarcoidosis was further confirmed by surface-enhanced laser desorption ionization time-of-flight mass spectrometry $[25,26]$. Another interesting finding was that the increase in SAA concentrations in sarcoidosis patients is associated with a decrease in paraoxonase 1 , an enzyme involved in HDLmediated antioxidant and anti-inflammatory functions. Paraoxonase 1 dysfunction seems to follow the granulomatous inflammation and oxidative stress typical of sarcoidosis [27].

A list of proteins identified by lipomics and involved in lipid metabolism and sarcoidosis pathogenesis has been included in Figure 1.

By combining proteomic results with genomic and transcriptomic findings, a research group identified some candidate genes (SERPINB1, FABP4, S100A8, HBEGF, IL7R, LRIG1, PTPN23, DPM2, and NUP214) linked to disease susceptibility and immune regulation, apoptosis, inhibition of protease activity, and lipid metabolism [28].

\section{Chitotriosidase in Atherosclerosis and Sarcoidosis}

Sarcoidosis and atherosclerosis are both inflammatory diseases in which activated macrophages are crucial because they release proinflammatory mediators, such as chitotriosidase. Atherosclerosis is associated with accumulation of lipids in artery walls after deposition of LDL cholesterol and fibrous matrix. In the early stage of this disease, monocytes differentiate into foam cells (lipidloaded macrophages generated by massive uptake of modified LDL) that take part in inflammatory responses 
Fig. 2. Human chitinase 1 (chitotriosidase) is a marker of activated macrophages involved in pulmonary sarcoidosis and in atherosclerosis.

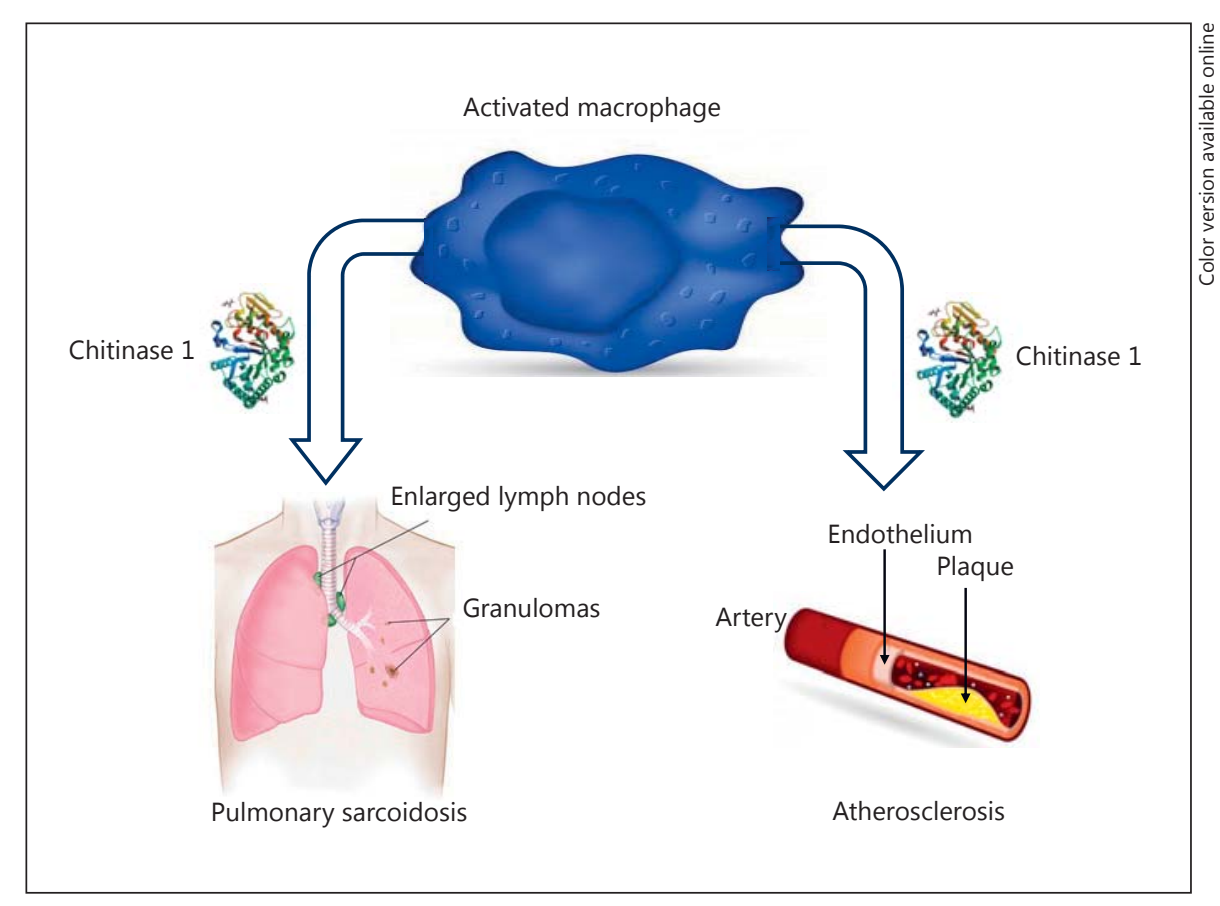

and tissue remodeling in the arterial intima (vessel lumen narrowing and intimal thickening associated with increased risk of thrombosis) [29-32]. Lipid accumulation promotes gene expression in macrophages, affecting the inflammatory process that occurs in atherogenesis [33]. Higher concentrations of chitotriosidase have been reported in the serum of patients with atherosclerosis than in controls (55-fold increase) as well as in sarcoidosis patients [29-32]. Chitinase 1 plays a role as inflammatory and profibrotic molecule in both diseases, suggesting that it may be a useful disease biomarker and indicator of atherosclerotic plaque formation, as well as in pulmonary granulomatous inflammation [34-36]. Human chitotriosidase concentrations, related to lipid-laden macrophages in human atherosclerotic vessel walls [29], generally increase with age and depend on accumulation of lipidfilled macrophages during progression of atherosclerosis $[37,38]$. Macrophage accumulation localized in the supra-aortic and coronary vessels is associated with increased serum CHIT1 activity, which reflects the state of activation of macrophages [29] (Fig. 2). Average CHIT1 activity in serum remained constant after 6 months of cholesterol- and triglyceride-lowering treatment with atorvastatin or bezafibrate, suggesting that LDL cholesterol and triglyceride reduction obtained with both drugs did not modify macrophage CHIT1 expression/activity in these subjects. Correction of the plasma lipid profile does not seem to affect the CHIT1 expression level in vivo, supporting the idea that CHIT1 activity cannot be used to monitor progression of atherosclerosis [38-43].

In conclusion, lipid metabolism seems to be altered in sarcoidosis and to contribute to disease pathogenesis. Sarcoidosis and atherosclerosis share several inflammatory mechanisms and biomarkers, such as chitotriosidase which is considered a reliable bioindicator of macrophage activity in both diseases.

Lipid alterations are related to oxidative stress and granulomatous inflammation. Patients with sarcoidosis show alterations in several cardiovascular biomarkers, overexpression of proteins involved in HDL and fatty acid metabolism, and cholesterol trafficking, increasing the risk of developing atherosclerosis.

\section{Disclosure Statement}

All authors have no conflict of interest to declare related to this topic.
Bargagli/Rosi/Pistolesi/Lavorini/ Voltolini/Rottoli 


\section{References}

1 Hunninghake GW, Costabel U, Ando M, et al: ATS/ERS/WASOG statement on sarcoidosis. Sarcoidosis Vasc Diffuse Lung Dis 1999;16: 149-173.

2 Agostini C, Trentin L, Facco M, et al: Role of IL-15, IL-2, and their receptors in the development of T cell alveolitis in pulmonary sarcoidosis. J Immunol 1996;157:910-918.

3 Prior C, Knight RA, Herold M: Pulmonary sarcoidosis: patterns of cytokine release in vitro. Eur Respir J 1996;9:47-53.

4 Kouranos V, Jacob J, Wells AU: Severe sarcoidosis. Clin Chest Med 2015;36:715-726.

5 Mochizuki I, Kubo K, Hond T: Widespread heavy damage of capillary endothelial cells in the pathogenesis of sarcoidosis - evidence by monoclonal von Willebrand factor immunohistochemistry in the bronchus and lung of patients with sarcoidosis. Sarcoidosis Vasc Diffuse Lung Dis 2014;31:182-190.

6 Schulman JM, LeBoit PE: Adipophilin expression in necrobiosis lipoidica, granuloma annulare, and sarcoidosis. Am J Dermatopathol 2015;37:203-209.

7 Mochizuki I, Kubo K, Honda T: Relationship between mitochondria and the development of specific lipid droplets in capillary endothelial cells of the respiratory tract in patients with sarcoidosis. Mitochondrion 2011;11: 601-606.

8 Tuleta I, Pingel S, Biener L, et al: Atherosclerotic vessel changes in sarcoidosis. Adv Exp Med Biol 2016;910:23-30.

9 Simonen P, Lehtonen J, Gylling H, et al: Cholesterol metabolism in cardiac sarcoidosis. Atherosclerosis 2016;248:210-215.

10 Bargagli E, Mazzi A, Rottoli P: Markers of inflammation in sarcoidosis: blood, urine, BAL, sputum, and exhaled gas. Clin Chest Med 2008;29:445-458.

11 Rahman I, Biswas SK, Kode A: Oxidant and antioxidant balance in the airways and airway diseases. Eur J Pharmacol 2006;533:222-239.

12 Psathakis K, Papatheodorou G, Platani M, et al: 8-Isoprostane, a marker of oxidative stress, is increased in expired breath condensate of patients with pulmonary sarcoidosis. Chest 2004;125:1005-1011.

13 Ivanišević J, Kotur-Stevuljević J, Stefanović A, et al: Dyslipidemia and oxidative stress in sarcoidosis patients. Clin Biochem 2012;45:677682.

14 Piotrowski WJ, Kurmanowska Z, Antczak A, et al: Exhaled 8-isoprostane as a prognostic marker in sarcoidosis. A short term followup. BMC Pulm Med 2010;10:23.

15 Bargagli E, Penza F, Vagaggini C, et al: Analysis of carbonylated proteins in BAL of patients with diffuse lung diseases. Lung 2007;185: 139-144.

16 Rottoli P, Magi B, Cianti R, et al: Carbonylated proteins in BAL of patients with sarcoidosis, pulmonary fibrosis associated with systemic sclerosis and idiopathic pulmonary fibrosis. Proteomics 2005;5:2612-2618.
17 Malli F, Bardaka F, Tsilioni I, et al: 8-Isoprostane levels in serum and bronchoalveolar lavage in idiopathic pulmonary fibrosis and sarcoidosis. Food Chem Toxicol 2013;61:160163.

18 Carleo A, Bennett D, Rottoli P: Biomarkers in sarcoidosis: the contribution of system biology. Curr Opin Pulm Med 2016;22:509-514.

19 Landi C, Bargagli E, Carleo A, et al: A functional proteomics approach to the comprehension of sarcoidosis. J Proteomics 2015; 128:375-387.

20 Bargagli E, Magi B, Olivieri C, et al: Analysis of serum amyloid A in sarcoidosis patients. Respir Med 2011;105:775-778.

21 Zhang Y, Chen X, Hu Y, et al: Preliminary characterizations of a serum biomarker for sarcoidosis by comparative proteomic approach with tandem-mass spectrometry in ethnic Han Chinese patients. Respir Res 2013; 14:18.

22 Salazar A, Pintó X, Mañá J: Serum amyloid A and high-density lipoprotein cholesterol: serum markers of inflammation in sarcoidosis and other systemic disorders. Eur J Clin Invest 2001;31:1070-1077.

23 Salazar A, Mañá J, Pintó X, et al: Corticosteroid therapy increases HDL-cholesterol concentrations in patients with active sarcoidosis and hypoalphalipoproteinemia. Clin Chim Acta 2002;320:59-64.

24 Salazar A, Maña J, Fiol C, et al: Influence of serum amyloid $A$ on the decrease of high density lipoprotein-cholesterol in active sarcoidosis. Atherosclerosis 2000;152:497-502.

25 Bons JA, Drent M, Bouwman FG, et al: Potential biomarkers for diagnosis of sarcoidosis using proteomics in serum. Respir Med 2007; 101:1687-1695.

26 Myioshi S, Hamada H, Kadowaki T, et al: Comparative evaluation of serum markers in pulmonary sarcoidosis. Chest 2010;137: 1391-1397.

27 Ivanišević J, Kotur-Stevuljević J, Stefanović A, et al: Association of serum amyloid A and oxidative stress with paraoxonase 1 in sarcoidosis patients. Eur J Clin Invest 2016;46:418-424.

28 Mayer A, Medica I, Peterlin B: Search for sarcoidosis candidate genes by integration of data from genomic, transcriptomic and proteomic studies. Med Sci Monit 2009;15:SR22SR28.

29 Malaguarnera L: Chitotriosidase: the yin and yang. Cell Mol Life Sci 2006;63:3018-3029.

30 Di Rosa M, Malaguarnera L: Chitotriosidase: a new inflammatory marker in diabetic complications. Pathobiology 2016;83:211-219.

31 Bargagli E, Maggiorelli C, Rottoli P: Human chitotriosidase: a potential new marker of sarcoidosis severity. Respiration 2008;76:234238.
32 Bargagli E, Bennett D, Maggiorelli C, et al: Human chitotriosidase: a sensitive biomarker of sarcoidosis. J Clin Immunol 2013;33:264270.

33 Langsted A, Freiberg JJ, Nordestgaard BG: Extent of undertreatment and overtreatment with cholesterol-lowering therapy according to European guidelines in 92,348 Danes without ischemic cardiovascular disease and diabetes in 2004-2014. Atherosclerosis 2016; 257:9-15.

34 Elmonem MA, van den Heuvel LP, Levtchenko EN: Immunomodulatory effects of chitotriosidase enzyme. Enzyme Res 2016; 2016:2682680.

35 Di Rosa M, Distefano G, Zorena K, Malaguarnera L: Chitinases and immunity: ancestral molecules with new functions. Immunobiology 2016;221:399-411.

36 Cho SJ, Weiden MD, Lee CG: Chitotriosidase in the pathogenesis of inflammation, interstitial lung diseases and COPD. Allergy Asthma Immunol Res 2015;7:14-21.

37 Rufo J, Taborda-Barata L, Lourenço O: Serum biomarkers in elderly asthma. J Asthma 2013; 50:1011-1019.

38 Canudas J, Cenarro A, Civeira F, et al: Chitotriosidase genotype and serum activity in subjects with combined hyperlipidemia: effect of the lipid-lowering agents, atorvastatin and bezafibrate. Metabolism 2001;50:447450.

39 Korolenko T, Johnston TP, Lykov AP, et al: A comparative study of the hypolipidaemic effects of a new polysaccharide, mannan Candida albicans serotype $\mathrm{A}$, and atorvastatin in mice with poloxamer 407-induced hyperlipidaemia. J Pharm Pharmacol 2016;68:15161526.

40 Korolenko TA, Pisareva EE, Filyushina EE, et al: Serum cystatin $\mathrm{C}$ and chitotriosidase in acute P-407 induced dyslipidemia: can they serve as potential early biomarkers for atherosclerosis? Exp Toxicol Pathol 2015;67:459466.

41 Güçlü A, Yilmaz MI, Tokmak TT, et al: Chitotriosidase as a novel biomarker of early atherosclerosis in hemodialysis patients. Hemodial Int 2017;21:41-46.

42 Azarsiz E, Karaca N, Levent E, et al: Chitotriosidase enzyme activity: is this a possible chronic inflammation marker in children with common variable immunodeficiency and early atherosclerosis? Ann Clin Biochem 2017, Epub ahead of print.

43 Pisareva EE, Goncharova IA, Tuzikov FV, et al: Role of changes in serum chitotriosidase activity in mice under conditions of hyperlipidemia and lipid-lowering effect of carboxymethylated (1-3)- $\beta$-D-glycan. Bull Exp Biol Med 2014;157:555-559. 